\title{
Lunar ISRU Energy Storage and Electricity Generation
}

\author{
Mario F. Palos ${ }^{\star}$, Pol Serra ${ }^{\star}$, Sonia Fereres ${ }^{\star \star}$, Keith Stephenson ${ }^{\star \star \star}$, Ricard González-Cinca S $^{\star}$ \\ ${ }^{\star}$ Universitat Politècnica de Catalunya - BarcelonaTech \\ Carrer d'Esteve Terradas 7, 08860 Castelldefels, Barcelona

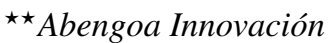 \\ C/ Energía Solar 1, Campus Palmas Altas, Sevilla (Spain) \\ $\star \star \star$ European Space Agency, ESTEC TEC-EPM \\ Keplerlaan 1, 2201AZ Noordwijk (The Netherlands) \\ †Corresponding author: ricard.gonzalez@upc.edu (Ricard González-Cinca)
}

\begin{abstract}
The survival of the astronauts and their equipment is the priority for any long-term exploration mission to the Moon. The provision of energy during the long lunar nights is a critical part of these missions. Several approaches have recently been considered to store and provide energy on the Moon by means of ISRU (In-Situ Resource Utilisation). We present a review of the energy requirements for a long mission scenario, and a trade-off analysis of the potentially suitable technologies for an ISRU-based system able to store heat and generate electricity. The most promising combinations of technologies are presented.
\end{abstract}

\section{Introduction}

Humankind tested its capacity to survive on the surface of the Moon for short periods of time in the Apollo missions almost 50 years ago. Since then, robotic missions to the Moon have spent several days and nights in the satellite. However, many technological challenges arise when planning a lunar (robotic and/or manned) mission fully operational during the night. Among these challenges, the need of a power supply system for both day and night remains open. Such system would ideally be based on ISRU, which would benefit its sustainability and allow lunar habitats a certain level of independence from systems brought from Earth.

The harsh lunar environment presents unique challenges for human exploration, such as the 14 Earth-day diurnal period in the equator, hard vacuum (approximately 10-11 torr), severe temperature day-night cycling, no atmospheric protection from meteor impacts, no magnetic protection against hard radiation, little amount of water, lunar dust.

Daytime in the equator of the Moon is about 14.77 Earth's days long, half of its synodic period. This makes the conventional method for power generation in space (solar panels plus batteries) inconvenient because a large amount of batteries is required. Moving away from the equator would only slightly modify these conditions, because the small axial tilt of the Moon ( $1.54^{\circ}$ to the ecliptic, $6.68^{\circ}$ to its orbital plane) results in minimal seasonal variations. Certain features of the Moon offer special illumination conditions, such as lava tubes, peaks of eternal light, and craters of eternal darkness. In these regions there is a permanent or almost permanent illumination or shadow, characteristics that can be exploited for energy generation or excess power dissipation.

The surface of the Moon, devoid of an atmosphere, experiences very large temperature oscillations. Simulations performed by Vasavada et al. ${ }^{22}$ show temperatures of $400 \mathrm{~K}$ during daytime and below $120 \mathrm{~K}$ during nighttime at the equator, with a decrease in the maximum temperature with latitude. Moreover, $0.5 \mathrm{~m}$ below the equator the temperature remains nearly constant over time at approximately $250 \mathrm{~K}$. Therefore, a few tens of centimeters of lunar regolith could effectively isolate humans and equipment from the variations of temperature above.

The lack of atmosphere has conditioned the surface of the Moon, which is covered by lunar regolith, a mantle of pulverized rock result of eons of bombardment by interplanetary matter of all sizes and energies. The density of the surface layer changes with depth and ranges from 1300 to $1900 \mathrm{~kg} / \mathrm{m}^{3},{ }^{20}$ and the density of the rocks underneath ranges from 2500 to $3400 \mathrm{~kg} / \mathrm{m}^{3}{ }^{13}$ both of them depending on the location. The composition of the soil is slightly different in the Highlands and in the Maria, the two distinct regions of the Moon. The regolith and rock samples brought to Earth in the Apollo missions are limited and have been affected by contact with the Earth's atmosphere. All measurements and characterization of materials since the seventies have been taken from orbit, by small mobile laboratories on board of surface rovers, or have been performed on terrestrial rocks considered sufficiently similar to those on the Moon. Table 1 shows the thermophysical properties of the regolith and rock layers. Raw regolith can in principle be considered to 
store sensible heat in a power generation system. The main advantages of raw regolith are ISRU, large availability, and large operating temperature range. The main disadvantages for the use of raw regolith as a thermal energy storage material are its low thermal conductivity, the need of a heat transfer fluid to transfer heat effectively, and the dispersed particle size, which may require compaction.

Table 1: Properties of lunar regolith and rock

\begin{tabular}{lcc}
\hline & Regolith & Rock \\
\hline Density $\left(\mathrm{kgm}^{-3}\right)$ & $1700^{(20)}$ & $2900^{(13)}$ \\
Thermal conductivity $\left(\mathrm{Wm}^{-1} \mathrm{~K}^{-1}\right)$ & $7.0 \cdot 10^{-3}(8)$ & 0.66 \\
Thermal diffusivity $\left(\mathrm{m}^{2} \mathrm{~s}^{-1}\right)$ & $6.86 \cdot 10^{-3}$ & $3.5 \cdot 10^{-7}(11)$ \\
Specific heat capacity $\left(\mathrm{Jkg}^{-1} \mathrm{~K}^{-1}\right)$ & $600^{(9)}$ & $650^{(9)}$ \\
\hline
\end{tabular}

Processing raw regolith by sintering could enhance its thermal properties so that the final product becomes more adequate for a thermal energy storage system. ${ }^{1,5}$ In a sintering process, a solid mass of material is compacted and formed by applying pressure or heat at temperatures below the liquefaction point. The most likely regolith sintering methods to be used on the Moon are based on microwaves, concentrated solar energy, or laser. In microwave sintering, the surface of powdered regolith is treated with specific microwave frequencies, with the objective of coupling the microwave energy with some of the constituents of regolith and melting them while other components remain solid. This method controls the elements that couple with the microwave energy, the depth of penetration, and the properties of the final product, by tuning the microwaves. Lunar regolith could be melted down to 0.5 meters and used to produce several types of structural materials, like bricks or solid pavement. ${ }^{21}$ Regolith sintering is also possible by concentrating solar power on top of the material. ${ }^{10}$ This method can achieve very high temperatures without the need of an electrical power source. However, processing is limited to daytime and requires a tracking to move the focal point. Moreover, sintering with this method only reaches depths of the orders of millimeters. Laser sintering allows a better control than the previous methods, both in intensity of the light and geometrical accuracy of the beam. Laser can also produce much higher temperatures than any other method, and achieve melting instead of sintering. While a sintered piece keeps some of the original grains intact, held together by other grains that have melted, selective laser melting can join all the components into an amorphous structure. ${ }^{6}$

Thermal Wadis are engineered solar energy storage systems that use modified regolith as a thermal storage mass. ${ }^{1}$ Wadis can store heat during the lunar day, and supply heat during the lunar night to rovers. They are good candidates to provide the required thermal energy for the survival of rovers and other equipment during periods of darkness. However, temperatures reached in a Wadi heated with a reflector and with a heat-loss protection are not high enough to run a heat engine efficiently during the lunar night. ${ }^{5}$ Therefore, alternative systems are required for missions with high power requirements.

We present a comparative analysis of ISRU-based power systems potentially suitable for lunar habitats. A review of power requirements for different lunar habitats is presented in Section 2. Section 3 contains a trade-off analysis of several for the subsystems and the full energy storage and electricity generation system. Conclusions are presented in Section 4.

\section{Power requirements for a lunar habitat}

Fig. 1 shows the relation between the mission objectives, energy requirements and power generation and storage systems for missions on the Moon. The energy requirements (which can be thermal and/or electrical) of a lunar mission are determined by several factors such as the landing site, lunar environment, span and profile of the missions, and whether it is robotic and/or manned. The energy requirements include the needs of both power generation and storage. There are several technological candidates for these two functions.

Since the last Apollo mission ended, the next manned mission to the Moon has always been in the horizon but has never happened. However, a trail of scientific and technical studies have been carried out with the aim at envisioning how to build a habitat, how to power it, and the power requirements. An analysis of these studies allows us to provide here an estimation of the power requirements for future lunar habitats. We assume in the analysis that the power system needs to be able to provide $100 \%$ of the required power.

Petri et al. ${ }^{18}$ proposed a settlement built in three progressive phases. The first one consists of an outpost with a power consumption of $25 \mathrm{kWe}$ (daytime, D) and $12 \mathrm{kWe}$ (nighttime, N) followed by an expansion reaching $80 \mathrm{kWe}$ (D) and $50 \mathrm{kWe}(\mathrm{N})$. The difference in day and night power is explained by the fact that the power generation capabilities are reduced at night. The third phase includes a larger habitat and the construction of ISRU facilities that would raise the 


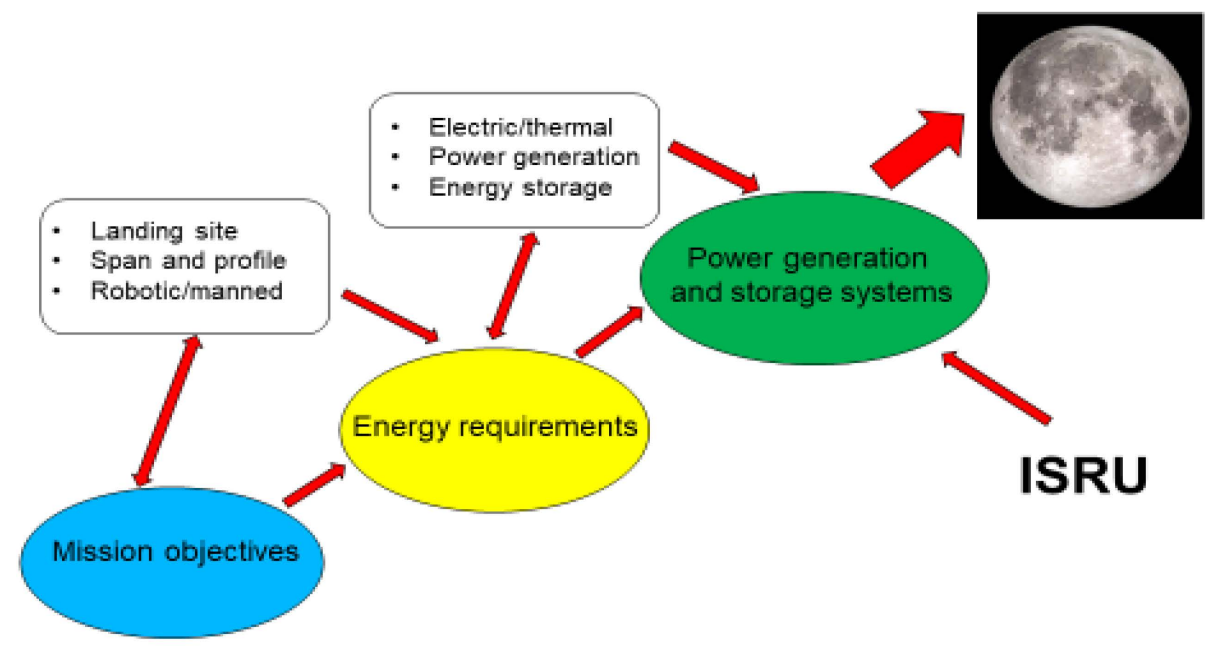

Figure 1: Relationship between mission objectives, energy requirements and power generation and storage systems for missions on the Moon.

consumption to $180 \mathrm{kWe}(\mathrm{D})$ and $150 \mathrm{kWe}(\mathrm{N})$. Photovoltaic cells (PV) are proposed during the day and regenerative fuel cells (RFC) during the night for the first phase, and a nuclear power plant and a pilot lunar liquid oxygen plant for later stages. Cataldo and Bozek ${ }^{4}$ described a 45-days mission to a lunar outpost that includes a preparation phase of the settlement using robots. Power consumption is estimated to be $12 \mathrm{kWe}(\mathrm{D})$ and $11 \mathrm{kWe}(\mathrm{N})$. Mason et al. ${ }^{16}$ analyzed the feasibility of fission nuclear power sources on the Moon. Although nuclear power is currently not being considered for manned missions, their estimations on power consumption are worthy for our analysis. The authors suggest installing a 5 -year lifespan $30 \mathrm{kWe}$ plant, and replacing it later on by two new plants producing $40 \mathrm{kWe}$ each. Khan et al. ${ }^{12}$ studied a power supply and storage system for a polar lunar base, consisting of PV and RFC, and discussed the use of batteries. A consumption of $81 \mathrm{kWe}$ is estimated without taking into account night conditions. Landis ${ }^{15}$ reviewed a large number of scenarios to provide power to a lunar base during the night. Power requirements of $100 \mathrm{kWe}$ (D) and $50 \mathrm{kWe}(\mathrm{N})$ are estimated. Balint ${ }^{2}$ analyzed several power generation systems for the Moon and Mars. The lunar settlement requirements are expected to gradually grow from a few kWe to $100 \mathrm{kWe}$. Later, ISRU facilities will add between $30 \mathrm{kWe}$ and $50 \mathrm{kWe}$.

We consider the Apollo program ${ }^{17}$ as a special case in the power requirements analysis. Six lunar modules successfully reached the surface of the Moon, and stayed there for durations between 21 and 72 hours. The lunar modules were initially designed to be powered by a combination of fuel cells and batteries. Three fuel cells could provide between $400 \mathrm{~W}$ and $1420 \mathrm{~W}$ each at 31 to $27 \mathrm{VDC}$. Therefore, the estimated theoretical maximum available power for the lander was $4.2 \mathrm{kWe}$, without taking batteries into account. Nevertheless, the fuel cells were removed from the power design shortly before the missions. The final version of the lunar module was powered by seven batteries (six initially, plus one that was added after the Apollo 13 accident). Five 400 Ah batteries where located in the descent section and two $300 \mathrm{Ah}$ batteries were in the ascent section. They all provided 28 VDC to the bus. Although the power consumption of the lunar module is not available, an estimation can be performed. Assuming a mission duration of 75 hours and a constant power consumption during this time, the batteries could provide a maximum of $970 \mathrm{~W}$ to the module. Consumption during EVAs and resting periods of the astronauts would be lower, and higher during ascent and descent operations.

Table 2 shows a summary of the power consumption of lunar outpost missions at different stages. The power requirements in surface outposts and bases are expected to range from $25 \mathrm{kWe}$ to a few hundreds of kWe during the early build-up phases. As the base becomes fully operational with in-situ resource production and closed-loop life support, power requirements could approach $1 \mathrm{MW}$. The night power requirements considered in this work are of the order of $10 \mathrm{kWe}$ (stage 1). 
Table 2: Summary of power consumption of lunar habitats

\begin{tabular}{|c|c|c|c|c|c|c|}
\hline & \multicolumn{6}{|c|}{ Power consumption (kWe) and sources } \\
\hline Study & 1st stage & 2nd stage & Early source & 3rd stage & Next stages & Later source \\
\hline \multirow{2}{*}{ Petri et al. ${ }^{18}$} & $25(\mathrm{D})$ & $80(\mathrm{D})$ & \multirow{2}{*}{$\mathrm{PV} / \mathrm{RFC}$} & $180(\mathrm{D})$ & \multirow{2}{*}{$200+$} & $\mathrm{PV} / \mathrm{RFC}+$ \\
\hline & $12(\mathrm{~N})$ & $50(\mathrm{~N})$ & & $150(\mathrm{~N})$ & & Nuclear \\
\hline \multirow{2}{*}{ Cataldo and Bozek ${ }^{4}$} & 12(D) & \multirow[t]{2}{*}{ - } & \multirow{2}{*}{-} & \multirow[t]{2}{*}{ - } & \multirow{2}{*}{-} & \multirow[t]{2}{*}{ - } \\
\hline & $11(\mathrm{~N})$ & & & & & \\
\hline Mason et al. ${ }^{16}$ & 30 & 80 & Nuclear & - & - & Nuclear \\
\hline Khan et al. ${ }^{12}$ & - & $81(\mathrm{D})$ & $\mathrm{PV} / \mathrm{RFC}$ & & - & Nuclear \\
\hline \multirow{2}{*}{ Landis $^{15}$} & \multirow{2}{*}{-} & 100(D) & \multirow{2}{*}{ Undefined } & \multirow{2}{*}{$100+$} & \multirow{2}{*}{-} & \multirow{2}{*}{-} \\
\hline & & $50(\mathrm{~N})$ & & & & \\
\hline Balint $^{2}$ & \multicolumn{2}{|c|}{$10-100$} & PV/RTG & $100+$ & - & Others \\
\hline Apollo $^{17}$ & \multicolumn{2}{|c|}{0.97} & Batteries & - & - & - \\
\hline
\end{tabular}

\section{Heat storage and electricity generation}

\subsection{System architecture}

Fig. 2 shows the proposed model for the energy storage and electricity generation system based on the work by Climent et al..$^{5}$ The energy collected by the Solar Collector is transported to a Energy storage subsystem and, when it is needed, to a Heat-to-electricity conversion unit. The excess of heat from the conversion is tranported to a Heat rejection unit. The dotted line in Fig. 2 shows the option of a direct energy transfer to the converter without storing it, which accounts for the direct generation of electricity from sunlight during daytime.

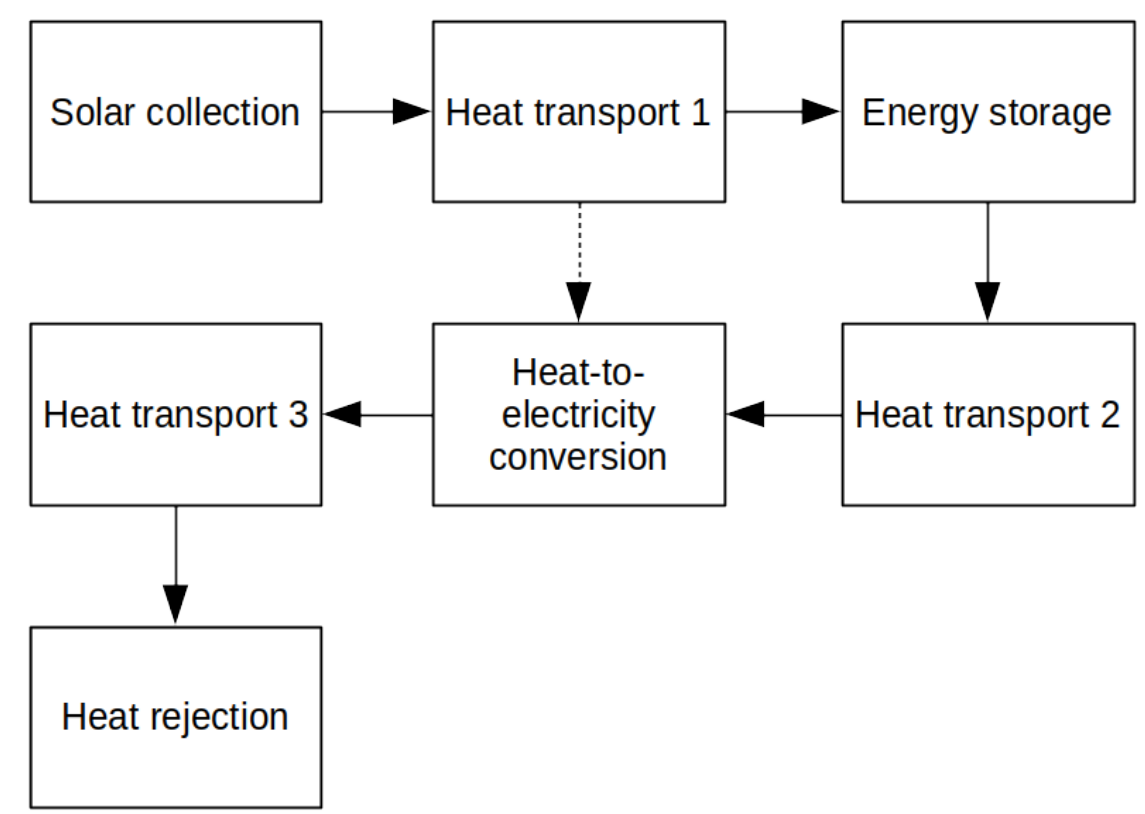

Figure 2: Model of the energy storage and electricity generation system. 


\subsection{Trade-off methodology}

A trade-off analysis of the identified technological options has been carried out for each subsystem and for the full system. Common criteria are defined for all the subsystems and specific criteria only for some of them. A weight is assigned to each criterion, which may differ in each subsystem, and technologies and components are scored for each criterion. Most of the scores for the criteria of the technologies in the different subsystems are assigned after a qualitative analysis. However, in some cases quantitative scoring rules have been used. The following criteria have been considered in the trade-off analysis of the subsystems.

Transport from Earth: Difficulty and cost of transport, including mass, storage volume, and ability to withstand the conditions of a rocket launch.

Installation and construction: complexity of construction and installation, as well as the associated risks.

Operation and maintenance: difficulty of operation, amount of human intervention required, complexity of repairs.

ISRU: amount of local materials used.

Scalability: feasibility of an expansion of capabilities of the subsystem.

Lifespan: expected duration of the component before repairs or replacement.

End of life: usefulness of the components after decommission, or possible hazards caused by the remains.

Cost: cost of development and operation.

TRL: Technology Readiness Level.

Technology maturity on Earth: stage of development, proven capabilities.

Operational in high/low temperatures: ability to operate, or at least survive, on specific extreme thermal conditions.

The following criteria have only been considered for some of the subsystems:

Concentration ratio: ratio between the collector aperture and the surface area of the receiver. Applicable to Solar energy collection.

Performance efficiency: power output to power input ratio. Applicable to Heat transport, Heat-to-electricity conversion, and Heat rejection.

Power, Specific power: total power generated and power-to-mass ratio. Applicable to Heat-to-electricity conversion.

Volumetric heat capacity, Thermal conductivity: thermal properties. Applicable to Energy storage (thermal masses) and Heat rejection.

All technologies receive a score between 0 and 5 in each criterion, except in the non-applicable (n/a) cases. The final score is calculated by means of:

$$
\text { Total }=\frac{\text { \#criteria }}{\# \text { applicable criteria }} \cdot \sum_{i}(\operatorname{score}(i) \cdot \text { weight }(i)) \text {, }
$$

where $i$ refers to each considered technology. Eq. 1 is meant to give a fair score to those technologies that are so different to their equivalents that do not fit in the trade-off, such as Direct illumination of thermal masses. If only a few criteria are applicable to a technology, but their scores are high in categories with high weight, the resulting score is very high. 
Table 3: Trade-off of solar energy collection technologies

\begin{tabular}{|c|c|c|c|c|c|c|c|}
\hline Evaluation criteria & Weight & 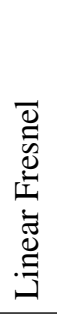 & 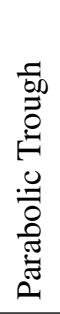 & 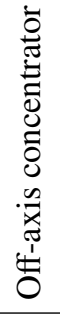 & 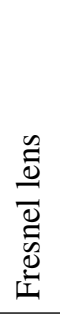 & 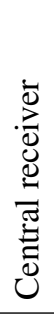 & 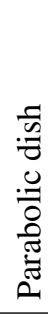 \\
\hline Transport from Earth & 3 & 3 & 3 & 2 & 1 & 3 & 3 \\
\hline Installation and construction & 2 & 3 & 3 & 4 & 4 & 2 & 3 \\
\hline Operation and maintenance & 2 & 4 & 4 & 3 & 3 & 2 & 2 \\
\hline ISRU & 5 & 0 & 0 & 0 & 0 & 0 & 0 \\
\hline Scalability & 3 & 4 & 3 & 4 & 3 & 4 & 3 \\
\hline Lifespan & 3 & 4 & 4 & 4 & 4 & 4 & 3 \\
\hline End of life & 1 & 2 & 2 & 2 & 2 & 2 & 2 \\
\hline Cost & 2 & 3 & 3 & 4 & 5 & 2 & 2 \\
\hline TRL & 1 & 4 & 4 & 4 & 4 & 4 & 4 \\
\hline Technology maturity on Earth & 2 & 5 & 5 & 5 & 5 & 5 & 5 \\
\hline Operational in high temperatures & 5 & 5 & 5 & 5 & 5 & 5 & 5 \\
\hline \multirow[t]{2}{*}{ Concentration ratio } & 2 & 2 & 3 & 2 & 4 & 4 & 5 \\
\hline & SCORE & 98 & 97 & 97 & 97 & 94 & 92 \\
\hline
\end{tabular}

\subsection{Solar collection}

Table 3 shows the outcome of the trade-off analysis of the technologies in the Solar energy collection subsystem. All the considered technologies belong to the category of Concentrated Solar Power (CSP). Instead of converting sunlight into electricity directly in the receiver, CSP plants concentrate sunlight using lenses or mirrors. The resulting heat is stored in a thermal battery or used to generate electricity directly from it. CSP plants, which are extensively used on Earth, are good candidates to perform better on the Moon due to the absence of atmosphere and a higher solar irradiance.

A first group of collectors to consider is composed of Parabolic dish (PD) and Central receiver (CR) plants, both designed for maximum concentration of sunlight. In PD plants sunlight is directed into their focal points with maximum theoretical concentration using paraboloid mirrors. Sun is tracked in two axes, pointing the center of the mirror to the Sun all day long. PD are big and complex structures that are difficult to build, transport, operate, and clean. A big moving mirror needs be at a sufficient distance from the ground in order to track the Sun without any obstacle, which results in a tall structure that would be difficult to reach by astronauts. Two axis tracking requires motors, and a parabolic mirror pointing in the wrong direction is useless. In addition, these systems are best suited for direct conversion to electricity in a heat engine located in the focus, as a fluid loop would be complex to set up in a system with a rotating base. This would be incompatible with the use of a thermal mass. CR plants also obtain high concentrations of sunlight. They consist in a central receiver tower, and a set of two-axis-tracking heliostats that focus the sunlight in the central receiver. Depending on the design and the power needs, the required number of mirrors can be very high, each of them having their own set of two-axis trackers. Moreover, the tower may need to be a tall structure, hard to transport, install and mantain.

A second category of collectors sacrifice concentration power in exchange of simplicity. Both Linear Fresnel reflectors (LFR) and Parabolic troughs (PT) concentrate sunlight in a line instead of a point, providing a more spread distribution of energy. The mirrors in these technologies are close to the ground and track the Sun in only one angle, if any. This makes the installation and repair simple, as the mirrors and motors are easily accessible. LFR consists in a field of flat mirrors configured to focus the light similarly to a huge parabolic cylinder mirror. An advantage of LFR is that individual flat mirrors are simple to fabricate and store. PT use sets of parabolic cylinder mirrors, which results in a better concentrating power than in LFR. The more complex shape of PT makes them more difficult to store. In addition, while in LFR systems multiple mirrors focus sunlight into a single collector, each PT mirror has its own collector.

We consider a third group of concentrators, which are characterized by their simplicity to build and operate. Nevertheless, they have a very little concentrating power and may be suitable only for low temperature scenarios. Off- 
axis concentrators consist in a static parabolic reflector and one or more Sun-tracking flat reflectors that guide sunlight into it. Fresnel lenses focus parallel light rays into a point similarly to a plano-convex lens, but with reduced mass. They are totally passive, easy to mantain and do not need to be in a tall and complicated structure, except for two-axis trackers. The low concentrating power could be improved with larger lenses, but there is an upper limit in the size of lenses before they start bending by their own weight. Fresnel lens are fragile, which makes the transport from Earth challenging.

In the trade-off analysis, technologies sharing a common collector (e.g. LFR) have been considered more scalable than those requiring new units to be built (e.g. PT). All collectors score zero points in ISRU since the production of mirrors and glass from regolith is being investigated, but has never been tested in lunar conditions and one can assume that it will not be available in the first settlements.

The outcome of the trade-off gives the highest score to the Linear Fresnel reflectors, closely followed by Parabolic troughs, Off-axis concentrators and Fresnel lenses. The technologies that are penalized for their complexity are rewarded for their performance, and vice-versa. The selection of a technology will depend on the factor considered to be more important for the mission, and the ability to reach the required temperatures for the thermal mass.

\subsection{Heat transport}

Table 4 shows the outcome of the trade-off analysis of the technologies in the Heat transport subsystem. The architecture of the heat storage and electricity generation system includes the heat transport in three connections of subsystems. The heat transport technology may differ in the three locations.

Table 4: Trade-off of heat transport technologies

\begin{tabular}{|c|c|c|c|c|c|c|c|}
\hline Evaluation criteria & Weight & 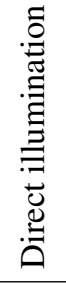 & 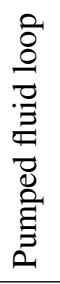 & 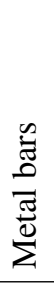 & 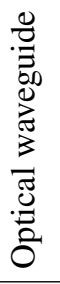 & 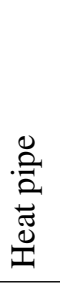 & 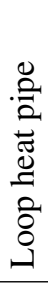 \\
\hline Transport from Earth & 3 & $\mathrm{n} / \mathrm{a}$ & 4 & 2 & 3 & 2 & 2 \\
\hline Installation and construction & 2 & 5 & 2 & 4 & 2 & 3 & 4 \\
\hline Operation and maintenance & 2 & 5 & 2 & 4 & 2 & 4 & 4 \\
\hline ISRU & 5 & $\mathrm{n} / \mathrm{a}$ & 0 & 0 & 0 & 0 & 0 \\
\hline Scalability & 2 & $\mathrm{n} / \mathrm{a}$ & 3 & 4 & 4 & 1 & 1 \\
\hline Lifespan & 2 & 5 & 4 & 5 & 5 & 5 & 5 \\
\hline End of life & 1 & $\mathrm{n} / \mathrm{a}$ & 2 & 4 & 2 & 2 & 2 \\
\hline Cost & 2 & 5 & 2 & 4 & 2 & 3 & 3 \\
\hline TRL & 1 & $\mathrm{n} / \mathrm{a}$ & 4 & 4 & 2 & 4 & 2 \\
\hline Technology maturity on Earth & 2 & $\mathrm{n} / \mathrm{a}$ & 4 & 5 & 4 & 4 & 2 \\
\hline Performance/Efficiency & 5 & 5 & 4 & 1 & 4 & 2 & 2 \\
\hline \multirow[t]{2}{*}{ Operational in high temperatures } & 5 & $\mathrm{n} / \mathrm{a}$ & 5 & 5 & 3 & 3 & 3 \\
\hline & SCORE & 156 & 97 & 96 & 86 & 77 & 73 \\
\hline
\end{tabular}

The Direct illumination method stands out because of its simplicity. Transmiting light from the collector to the thermal mass through the vacuum is simple, low cost, requires no maintenance nor transport of spare parts, and there is no energy lost in conversions. However, this method interfaces poorly with some of the collectors and all the thermal masses, as heating them from the top is not optimal. ${ }^{7}$ Moreover, this technology is only applicable for the first heat transport subsystem just after the collector.

A Pumped fluid loop is the most versatile option to transport heat. It allows several choices of heat transfer fluids, and fluid speed can be dinamically changed in order to adapt the heat transfer. This technology is well known both on Earth and in space (e.g. in the ISS ammonia loop). A main disadvantage is that installation and maintenance may require burying and digging up pipes and pumps when they are affected by wear and, depending on the transfer fluid, by corrosion.

Metal bars and Optical waveguides are two affordable, simple and passive options. The first one transports energy passively as sensible heat. The rates of thermal transmission depend on the available materials. Optical waveguides 


\section{LUNAR ISRU ENERGY STORAGE AND ELECTRICITY GENERATION}

transfer energy in the form of light with very little losses, but they suffer from similar interfacing problems with a thermal mass as the Direct illumination.

Heat pipes are passive systems that transport energy as latent heat, greatly improving the transfer rates. They are not effective over large distances and cannot fight against gravity, problems that are overcome by the Loop heat pipe design. Both are difficult to upgrade because their geometry is fixed at the moment of construction.

One of the advantages of Metal bars, Heat pipes and Loop heat pipes can become a disadvantage during the lunar night. They transport heat passively from the hot collector to the colder thermal mass during the day, without any need for operation. However, this lack of operation also implies that they cannot be disconnected. When the temperature gradient between the collector and the thermal mass is reversed during the lunar night, these devices will in principle transport heat from the thermal mass to the collector, dissipating it into space. Optical waveguides, while also passive, will not operate backwards.

Aside from the special mention for the Direct illumination approach, Pumped fluid loop and Metal bars are the best scored options. The first one is adequate for all three heat transport subsystems, while the Metal bars are best suited for the connection between the Heat-to-electricity conversion unit and the Heat rejection subsystem.

\subsection{Energy storage}

Table 5 shows the outcome of the trade-off analysis of the technologies in the Energy storage subsystem. All the considered technologies store energy in form of heat except the fuel cells with ISRU hydrogen, for which the system architecture in Fig. 2 would change.

Table 5: Trade-off of energy storage technologies

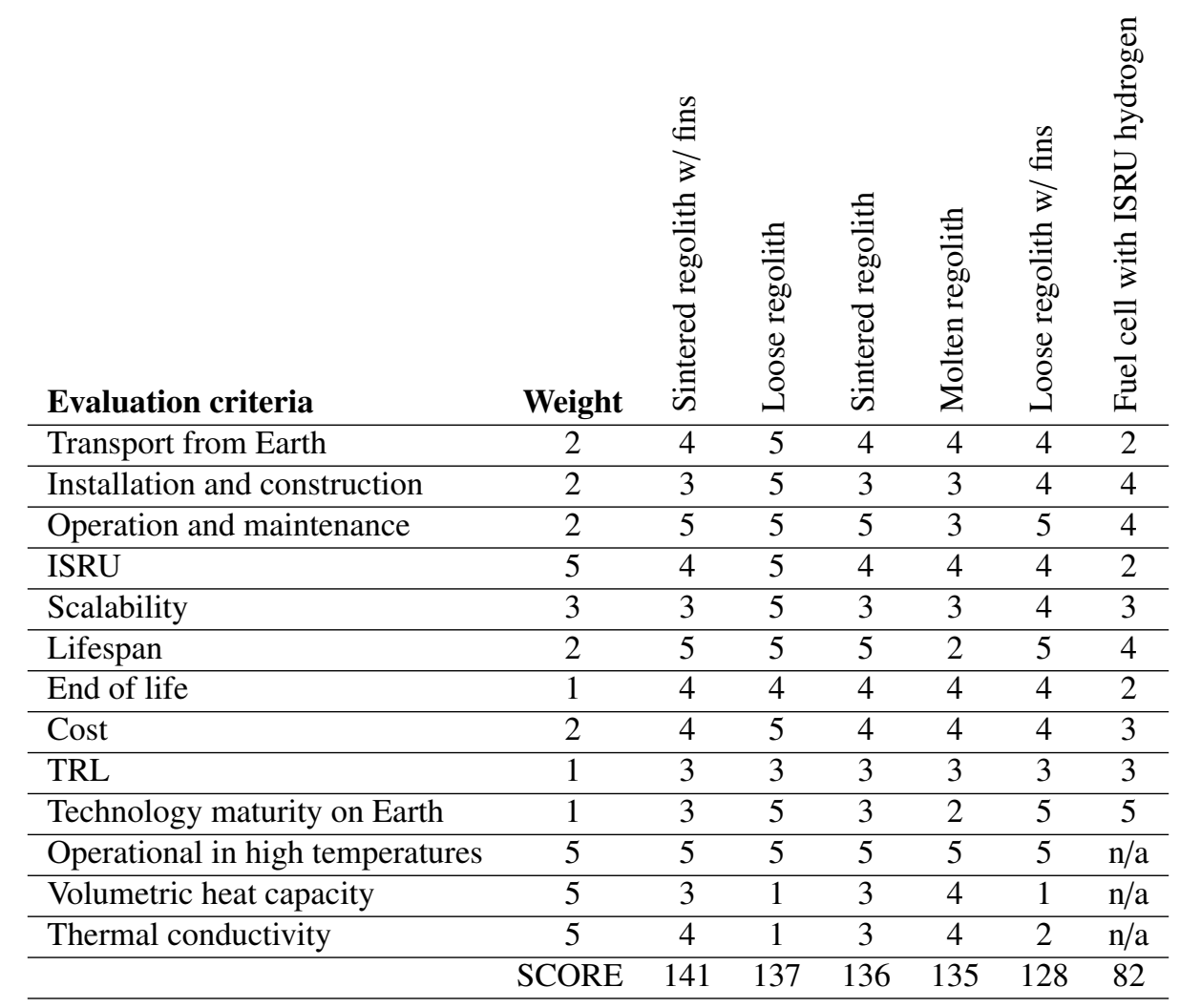

The Energy storage subsystem is the perfect candidate for satisfying the ISRU criterion of the power system. Raw or processed regolith can be converted into a heat storage device. Regolith is a costless component which is largely available for repairing or expanding the setup in the future. Moreover, it does not leave behind dangerous components, and the only equipment needed to be brought from Earth are pipes for the fluid transfer and devices for regolith processing in some of the options.

Loose regolith is ahead of the other options for an Energy storage subsystem in all the aforementioned aspects. It consists in raw lunar regolith surrounding a heat-exchanging structure, which could consist in pipes buried under the lunar surface. However, all these advantages are disputed by the poor thermal characteristics of loose regolith (Table 
1). Adding metal fins can greatly improve thermal conductivity, ${ }^{7}$ but it still lacks the capacity to store large amounts of energy.

Sintering of regolith can enhance the thermal properties of regolith while keeping its advantages. The use of metal fins would increase conductivity even further.

Molten regolith is in principle the best option in terms of energy density. While solid regolith blocks store energy as sensible heat, molten regolith would store it as latent heat after the phase change. Nevertheless, the problems and risks associated with melting regolith and moving a lava flow around the lunar base greatly affect the feasibility of this option.

The last option considered is the production of fuel cells with local water. Obtaining lunar water for the batteries would reduce the mass to be brought from Earth and make electrical storage of energy more feasible. However, fuel is only one part of a battery, and cases, controllers, and cables need to be brought as well. In addition, even if the presence of water in the Moon has been demonstrated, it is unclear where it is located and how much there is. The use of fuel cells would imply changes in the power system architecture, probably generating electrical energy directly from the concentrated solar power or photovoltaic panels.

The storage of energy in regolith of any kind has never been tested, neither on Earth nor on the Moon. Heat has been stored in concrete at DLR ${ }^{14}$ and at EnergyNest, ${ }^{3}$ although a generator never run for the time required in our application.

The best option obtained from the trade-off analysis of Energy storage technologies is sintered regolith with metal fins. If including fins inside the sintered block proves to be difficult, the sintered regolith option is close behind, as well as loose regolith and molten regolith, which could be used if their performances are proven to be sufficiently good.

\subsection{Heat-to-electricity conversion}

Table 6 shows the outcome of the trade-off analysis of the technologies in the Heat-to-electricity conversion subsystem.

Table 6: Trade-off of heat-to-electricity conversion technologies

\begin{tabular}{|c|c|c|c|c|c|c|c|c|}
\hline Evaluation criteria & Weight & $\stackrel{0}{\Xi}$ & 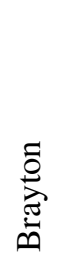 & 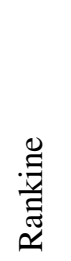 & 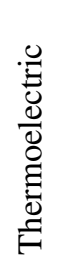 & 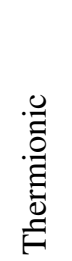 & 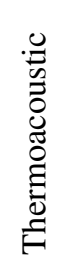 & 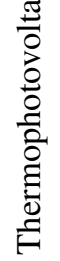 \\
\hline Transport from Earth & 4 & 3 & 2 & 1 & 1 & 1 & 3 & 1 \\
\hline Installation and construction & 2 & 2 & 2 & 2 & 4 & 4 & 2 & 4 \\
\hline Operation and maintenance & 2 & 4 & 3 & 1 & 5 & 5 & 4 & 5 \\
\hline ISRU & 5 & 0 & 0 & 0 & 0 & 0 & 0 & 0 \\
\hline Scalability & 3 & 3 & 3 & 3 & 5 & 5 & 3 & 5 \\
\hline Lifespan & 3 & 4 & 3 & 3 & 4 & 3 & 3 & 3 \\
\hline End of life & 1 & 2 & 2 & 2 & 2 & 2 & 2 & 2 \\
\hline Cost & 2 & 2 & 2 & 1 & 3 & 3 & 2 & 2 \\
\hline TRL & 1 & 4 & 4 & 2 & 5 & 5 & 1 & 2 \\
\hline Technology maturity on Earth & 2 & 4 & 4 & 4 & 3 & 2 & 1 & 2 \\
\hline Performance/Efficiency & 5 & 5 & 4 & 3 & 1 & 2 & 3 & 3 \\
\hline Power & 5 & 3 & 4 & 5 & 3 & 3 & 3 & 1 \\
\hline Specific power & 4 & 2 & 4 & 5 & 1 & 1 & 1 & 1 \\
\hline \multirow[t]{2}{*}{ Operational in high/low temperatures } & 5 & 5 & 5 & 5 & 5 & 5 & 5 & 5 \\
\hline & SCORE & 136 & 135 & 127 & 117 & 117 & 110 & 107 \\
\hline
\end{tabular}

Four heat engines have been considered in the analysis: Stirling, Brayton, Rankine, and Thermoacoustic. Thermoacoustic engines fall down in the ranking because, although they are very promising, up until now they have not been developed as much as the others.

Stirling, Brayton and Rankine are, in this order, increasingly complex and increasingly efficient. Rankine engine is the most efficient one thanks to the use of latent heat of the transfer fluid. The efficiency in these engines are one 


\section{LUNAR ISRU ENERGY STORAGE AND ELECTRICITY GENERATION}

order of magnitude above the other considered technologies. The heat engines are relatively heavy and bulky, and the presence of moving parts and flowing liquids or gases implies that repairs will be necessary at some point of their lifetime. Some of the engines have been tested in space (e.g. Stirling cyocoolers ${ }^{19}$ ), where issues with fluid flow in microgravity have been encountered. However, thanks to the lunar gravity, albeit small, their behaviour on the surface of the Moon could be predicted. A main disadvantage of heat engines is that they are not scalable, hence new engines should be brought from Earth in order to expand the system.

The other considered technologies (Thermoelectric, Thermionic and Thermoacoustic) are easy to expand. While each of them transforms energy by means of a different physical phenomenon, they all share several characteristics. They are small, low cost, passive and modular, with very little power per unit, but easy to install and form a big surface covering a thermal mass. Thermionic and thermoelectric generators have already been tested in small satellites. Although these technologies may seem to be ideal choices, their small specific power in the current state of development is a significant disadvantage. The number of devices that need to be brought from Earth is very large even for low power requirements.

Stirling, Brayton, and Rankine engines are the best scored technologies in the trade-off. Similar to the solar collectors, the technology with the best power generation is more complex and vice-versa, their scores ending up being very similar.

\subsection{Heat rejection}

Table 7 shows the outcome of the trade-off analysis of the technologies in the Heat rejection subsystem. Two main options have been considered for heat rejection: dumping the heat on the surface of the Moon, or into space by means of a radiator.

Table 7: Trade-off of heat rejection technologies

\begin{tabular}{|c|c|c|c|c|c|}
\hline Evaluation criteria & Weight & 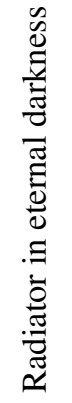 & 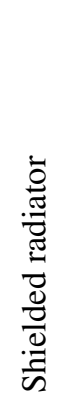 & 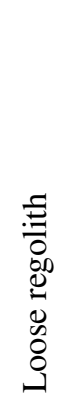 & 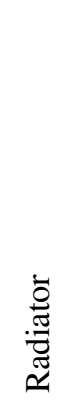 \\
\hline Transport from Earth & 2 & 2 & 2 & 5 & 2 \\
\hline Installation and construction & 2 & 2 & 3 & 4 & 4 \\
\hline Operation and maintenance & 2 & 5 & 5 & 5 & 5 \\
\hline ISRU & 5 & 3 & 3 & 5 & 0 \\
\hline Scalability & 3 & 4 & 4 & 5 & 4 \\
\hline Lifespan & 3 & 5 & 5 & 5 & 5 \\
\hline End of life & 1 & 3 & 3 & 5 & 3 \\
\hline Cost & 2 & 4 & 3 & 5 & 4 \\
\hline TRL & 1 & 5 & 5 & 3 & 5 \\
\hline Technology maturity on Earth & 2 & 5 & 5 & 5 & 5 \\
\hline Performance/Efficiency & 4 & 5 & 4 & 1 & 3 \\
\hline Operational in low temperatures & 5 & 5 & 5 & 5 & 5 \\
\hline Volumetric heat capacity & 4 & 3 & 3 & 1 & 3 \\
\hline \multirow[t]{2}{*}{ Thermal conductivity } & 4 & 4 & 4 & 1 & 4 \\
\hline & SCORE & 159 & 155 & 148 & 140 \\
\hline
\end{tabular}

The use of the Moon as a heat sink is convenient because it lowers the equipment needed, as radiators normally represent a big part of the weight of a thermal system. However, the thermal characteristics of this simple approach makes it a bad candidate for heat rejection.

The three types of radiators considered mainly differ in their location. Radiators could be built from the aluminum salvaged from the spent stages of a spacecraft, or from a metal obtained in-situ. Their performance relies on a low temperature, the cooler they are the better they perform. Thus, the presence of a shield greatly increases the efficiency. 
All the radiators have a similar cost and are passive (considering that the heat transfer fluid, if any, is part of the third Heat transport subsystem). Installation is reasonably easy for radiators, harder for shielded radiators (depending on the shield), and it may be very challenging inside craters, depending on their characteristics.

The radiator in a crater of eternal darkness gets the highest score in the trade-off. However, since its applicability is limited to certain regions in the poles, the second best option (shielded radiator) is more realistic for a larger number of landing sites.

\subsection{Discussion}

The trade-off analysis is a tool that gives broad information about the technologies under study, but it is not a perfect representation of them. For this reason, a technology cannot be discarded if the difference in score with the highest rated ones is small. Moreover, in the analysis of the full system, the interactions between connected subsystems must be taken into account. Two apparently ideal devices cannot be part of the full system if the interaction between them is poor or even impossible. In addition, and particularly for the present study, if there are no ISRU components in the system or if the system cannot provide the required power, it will be discarded.

More criteria related to logistics matters (such as size, weight, ease of transport, and cost) than to efficiency have been considered. Therefore, the analysis is biased towards simple technologies that may prove to be inefficient when the full system is considered in further simulation and experimental tests.

Attending only to the scores, the following combination of technologies would be the most recommendable:

Linear Fresnel reflectors $\rightarrow$ Direct illumination $\rightarrow$ Sintered regolith with fins $\rightarrow$ Pumped fluid loops $\rightarrow$ Stirling engine $\rightarrow$ Pumped fluid loop $\rightarrow$ Radiator in eternal darkness

For the second and third Heat transfer subsystem, the second best option (Pumped fluid) has been chosen, as the Direct illumination only makes sense for the first section. Linear Fresnel reflectors are the first choice for the solar energy collection, and they are somewhat with the Direct illumination heat transfer. However, the Direct illumination interfaces poorly with a regolith block. ${ }^{7}$ The inclusion of metal fins helps with the heat transfer, but the system as a whole would work better with either a different heat transfer method or a different heat storage technology. The heat engine and the cold part of the system can match well provided that the fluid used in the cold side can stand the lunar night temperature.

Alternative combinations of technologies with good scores and correct interfacing can be suggested. For example, Linear Fresnel reflectors would interface better with Pumped fluid loops, which can bring a constant supply of cold fluid to the focal line and carry the heat away towards a sintered regolith mass. Although an effective procedure for sintering large blocks of regolith has not yet been developed, the progress in the area is very promising and one can expect that by the time the lunar settlement will be built the enhanced thermal properties of a sintered regolith block will outweigh the troubles of building one. The Stirling engine, a simple system with space heritage and good performance, is the selected technology for energy conversion. Nevertheless, if passive converters, like thermoelectrics, improve sufficiently in the coming years, they will probably become the preferred solution. Finally, a radiator in eternal darkness is clearly the best option for heat rejection. However, given the limited number of locations where it can be used, it is more recommendable to consider a shielded radiator ideally built with in-situ materials. Therefore, the following set of components is proposed for the lunar ISRU energy storage and electricity generation system:

Linear Fresnel reflectors $\rightarrow$ Pumped fluid loop $\rightarrow$ Sintered regolith block with metal fins $\rightarrow$ Pumped fluid loop $\rightarrow$ Stirling engine $\rightarrow$ Pumped fluid loop $\rightarrow$ Shielded radiator

\section{Conclusions}

A trade-off analysis of the technologies and components that could be used in a lunar ISRU-based thermoelectric plant that fulfills the power requirements for settlement missions has been presented. The requirements have been established from the analysis of previous works and missions.

The comparative analysis has been carried out by establishing thresholds for an objective scoring when possible. However, in most of the criteria a qualitative evaluation has been performed. The outcome of the analysis provides a recommended system considering both the scores of the components and the interface between them. Nevertheless, there are still some uncertainties associated to some technologies. In addition, the score of some components are very similar. Therefore, the analysis performed does not define the system that should be built on the Moon but it allows us to discard some technologies and focus on the most promising ones. Further computational and experimental tests of the proposed system are highly recommended. 


\section{Acknowledgements}

Authors acknowledge the European Space Agency for financial support.

\section{References}

[1] R. Balasubramaniam, R. Wegeng, S. Gokoglu, N. Suzuki, and K. Sacksteder. Analysis of solar-heated thermal wadis to support extended-duration lunar exploration. In Proceedings of the 47th AIAA Aerospace Sciences Meeting (2009), volume AIAA 2009-1339, page 092407.

[2] T. S. Balint. Comparison of Power System Options Between Future Lunar and Mars Missions. In Proceedings of the International Lunar Conference 2005. Comparison of Power System Options Between Future Lunar and Mars Missions no. 818, 2005.

[3] P. G. Bergan and C. J. Greiner. A New Type of Large Scale Thermal Energy Storage. Energy Procedia, 58:152$159,2014$.

[4] R. L. Cataldo and J. M. Bozek. Power Requirements for the First Lunar Outpost (FLO). In Proceedings of the 10th Symposium on Space Nuclear Power and Propulsion. Tenth Symposium on Space Nuclear Power and Propulsion, 1993.

[5] B. Climent, O. Torroba, R. González-Cinca, N. Ramachandran, and M.D. Griffin. Heat storage and electricity generation in the Moon during the lunar night. Acta Astronautica, 93:352-358, 2014.

[6] M. Fateri and A. Gebhardt. Process Parameters Development of Selective Laser Melting of Lunar Regolith for On-Site Manufacturing Applications. International Journal of Applied Ceramic Technology, 12(1):46-52, 2015.

[7] Patrick Fleith, Aidan Cowley, Pablo López Córdoba, Rebecca Frank, Aarón Valle Lozano, Alberto Canals Pou, and Ricard González-Cinca. In-Situ Approach for Thermal Energy Storage and Thermoelectricity Generation on the Moon (submitted to Planetary and Space Science). 2019.

[8] Paul O. Hayne, Joshua L. Bandfield, Matthew A. Siegler, Ashwin R. Vasavada, Rebecca R. Ghent, Jean-Pierre Williams, Benjamin T. Greenhagen, Oded Aharonson, Catherine M. Elder, Paul G. Lucey, and David A. Paige. Global Regolith Thermophysical Properties of the Moon From the Diviner Lunar Radiometer Experiment: Lunar Regolith Thermophysical Properties. Journal of Geophysical Research: Planets, 122(12):2371-2400, December 2017.

[9] B.S. Hemingway, R.A. Robie, and W.H. Wilson. Specific heats of lunar soils, basalt, and breccias from the Apollo 14, 15, and 16 landing sites, between 90 and 350K. In Proceedings of the Fourth Lunar Science Conference (1973), volume 3, pages 2481-2487.

[10] P. E. Hintze, J. Curran, and T. Back. Lunar Surface Stabilization via Sintering or the Use or Heat Cured Polymers. pages 1009-1015. 47th AIAA Aerospace Science Meeting, 2009.

[11] K Horai and J.L. Winkler, Jr. Thermal diffusivity of two Apollo 11 samples, 10020,44 and 10065,23; Effect of petrofabrics on the thermal conductivity of porous lunar rocks under vacuum. In Proceedings of the 11th Lunar and Planetary Science Conference (1980), volume 3, pages 1777-1788.

[12] Z. Khan, A. Vranis, and B. Manners. Lunar Outpost: A Review of the Power Generation, Energy Storage, Power Management and Distribution (PMAD) System Requirements and Potential Technologies. Technical report, NASA, 2006.

[13] W. S. Kiefer, R. J. Macke, D. T. Britt, A. J. Irving, and G. J. Consolmagno. The Density and Porosity of Lunar Rocks. Geophysical Research Letters, 39(7), 2012.

[14] Doerte Laing, Wolf-Dieter Steinmann, Michael Fiß, Rainer Tamme, Thomas Brand, and Carsten Bahl. Solid Media Thermal Storage Development and Analysis of Modular Storage Operation Concepts for Parabolic Trough Power Plants. Journal of Solar Energy Engineering, 130(1), 2008.

[15] G. A. Landis. Solar Power for the Lunar Night. 9th Biennial SSI/Princeton Conference on Space Manufacturing, 1989. 
[16] L. Mason, D. Poston, and L. Qualls. System Concepts for Affordable Fission Surface Power. Space Technology and Applications International Forum (STAIF), 2008.

[17] NASA. Apollo Operations Handbook - Electrical Power System, 1969.

[18] D. A. Petri, R. L. Cataldo, and J. M. Bozek. Power System Requirements and Definition for Lunar and Mars Outposts. pages 18-27. Proceedings of the 25th Intersociety Energy Conversion Engineering Conference, vol. 1, 1990.

[19] R.G. Ross. JPL cryocooler development and test program: A 10-year overview. In 1999 IEEE Aerospace Conference. Proceedings (Cat. No.99TH8403), pages 115-124 vol.2, Snowmass at Aspen, CO, USA, 1999. IEEE.

[20] E. N. Slyuta. Physical and Mechanical Properties of the Lunar Soil (a Review). Solar System Research, 48(5):330$353,2014$.

[21] L. A. Taylor and T. T. Meek. Microwave Sintering of Lunar Soil: Properties, Theory, and Practice. Journal of Aerospace Engineering, 18:188-196, 2005.

[22] A. R. Vasavada, D. A. Paige, and S. E. Wood. Near-Surface Temperatures on Mercury and the Moon and the Stability of Polar Ice Deposits. Icarus, 141(2):179—193, 1999. 